\title{
Toward High-Intensity Ionization Chamber Beam Intensity Monitors in Switchyard
}

\author{
Adam Watts ${ }^{1, *}$ \\ ${ }^{1}$ Fermilab, Accelerator Division, External Beamlines \\ *awatts@fnal.gov
}

\section{ABSTRACT}

Beam intensity in the Switchyard beamlines is monitored by Secondary Emission Monitors ("SEMs") and lonization Chambers ("ICs"). While less-expensive and easier to calibrate than SEMs, IC performance has typically degraded at higher beam intensities. This paper describes a proof-of-principle modification to a standard IC and associated electronics to increase the upper dynamic range of the system, showing that it is feasible to use ICs for high-intensity beamlines.

\section{Introduction}

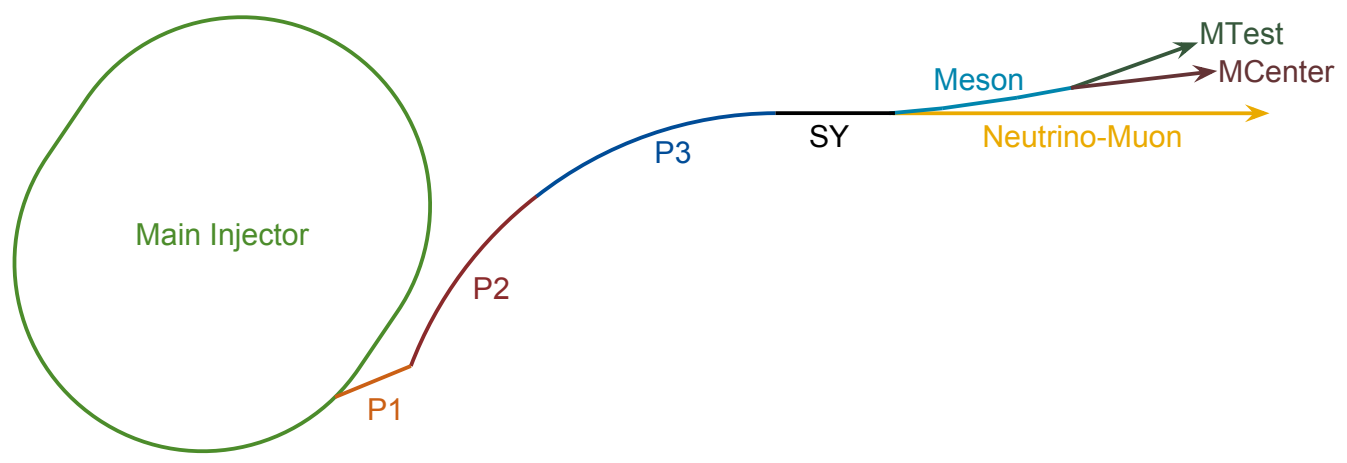

Figure 1. Overview of the Fermilab SY120 Fixed Target beam lines.

The Switchyard Fixed Target program (a.k.a. "SY120") currently supports up to three users: two Fermi Test Beam users in the Meson beamline, and the upcoming E1039 experiment in the Neutrino-Muon (NM) line. $120 \mathrm{GeV}$ proton beam is provided via resonant extraction from the Main Injector synchrotron, and thus the beam must be split twice to serve all three users simultaneously. The test beam users request a wide range of primary beam intensity, anywhere from 1E8 to 1E11 protons per pulse ("ppp"). The E1039 experiment will request upwards of 1E13 protons per pulse. Thus beam intensity monitors in the SY120 beamlines must be capable of five orders of magnitude dynamic range.

At the extreme low-end of intensities delivered in the Meson beamline, 1E8 protons per 4.2 second spill corresponds to an average beam current of 3.8 picoAmps, with average bunch occupancy of less than one proton per bucket. Detecting this beam for the purpose of integrating the total intensity per spill is very difficult with traditional beam current transformers used in higher-intensity beamlines and accelerators. Therefore, two types of beam intensity detectors have been used in the SY120 Fixed Target beamlines: ionization chambers ("ICs") and Secondary Emission Monitors ("SEMs"). The former have been used to detect per-spill intensity ranges of about $1 \mathrm{E} 7$ to $1 \mathrm{E} 11$, and the latter for $1 \mathrm{E} 9$ to $1 \mathrm{E} 13 .{ }^{1}$

The response of the ICs in the Switchyard beamlines decreases at beam intensity higher than about 1E11 ppp. Thus these chambers are used in lower-intensity beamlines where the detector response is linear over the operating intensity range. While SEMs have a linear response up to intensities of $1 \mathrm{E} 13 \mathrm{ppp}$, they are far more expensive than ICs and require regular recalibration as the signal foil secondary electron yield degrades with beam exposure. ${ }^{2}$ Furthermore, SEMs necessitate more material in the beam than ICs, which increases residual radiation and beam scattering. The successful use of ICs at higher beam intensities would save money on building new instrumentation, improve beam quality by reducing interaction with material, and save time in recalibrating detectors.

This paper focuses on identifying and alleviating sources of saturation in IC response for beam intensity above 1E12 ppp. 
In particular, signal saturation in both the physical IC detector itself, as well as the digitizing electronics that read the ionization current, are explored.

\section{Ion Chambers}

The basic principle of the ionization chamber is that beam particles pass through and ionize an inert gas. A high-voltage and ground plane on either side of the gas creates an electric field, causing the electron/ion pairs to drift apart and create a small ionization current, which is directly proportional to the intensity of the beam. The total beam intensity for that pulse is determined by integrating the ionization current. A model of this scenario is shown in Figure 2. The IC used for these studies

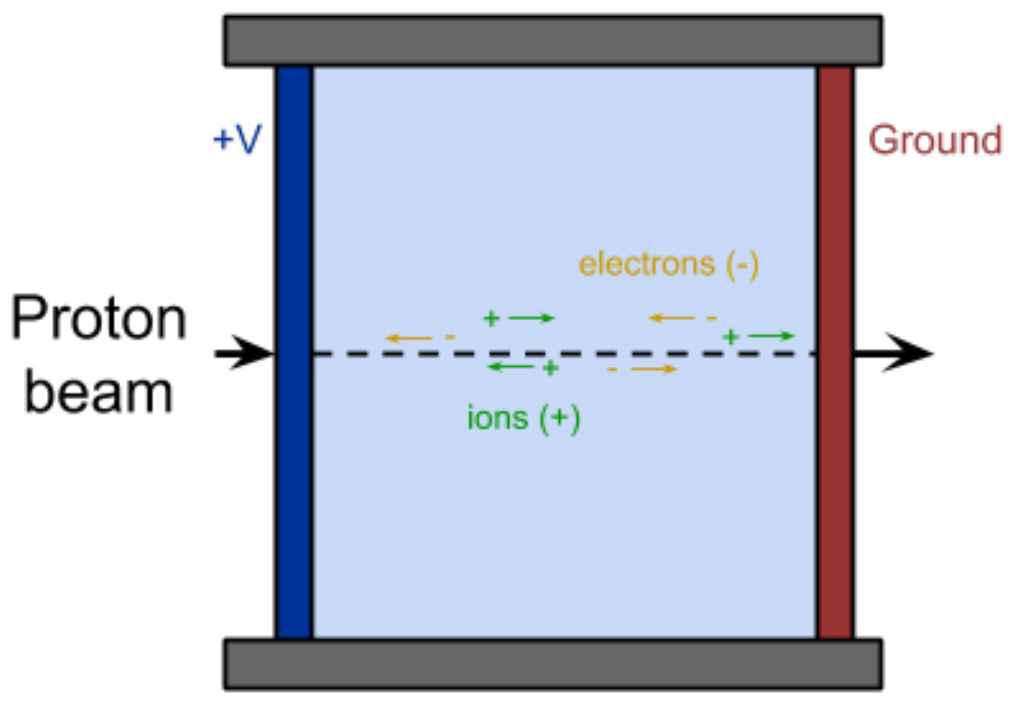

Figure 2. The basic principle of an ionization chamber: protons passing through an inert gas create electron/ion pairs, which are collected by the electric field generated from the potential between chamber walls, generating a small ionization current that is proportional to the beam intensity.
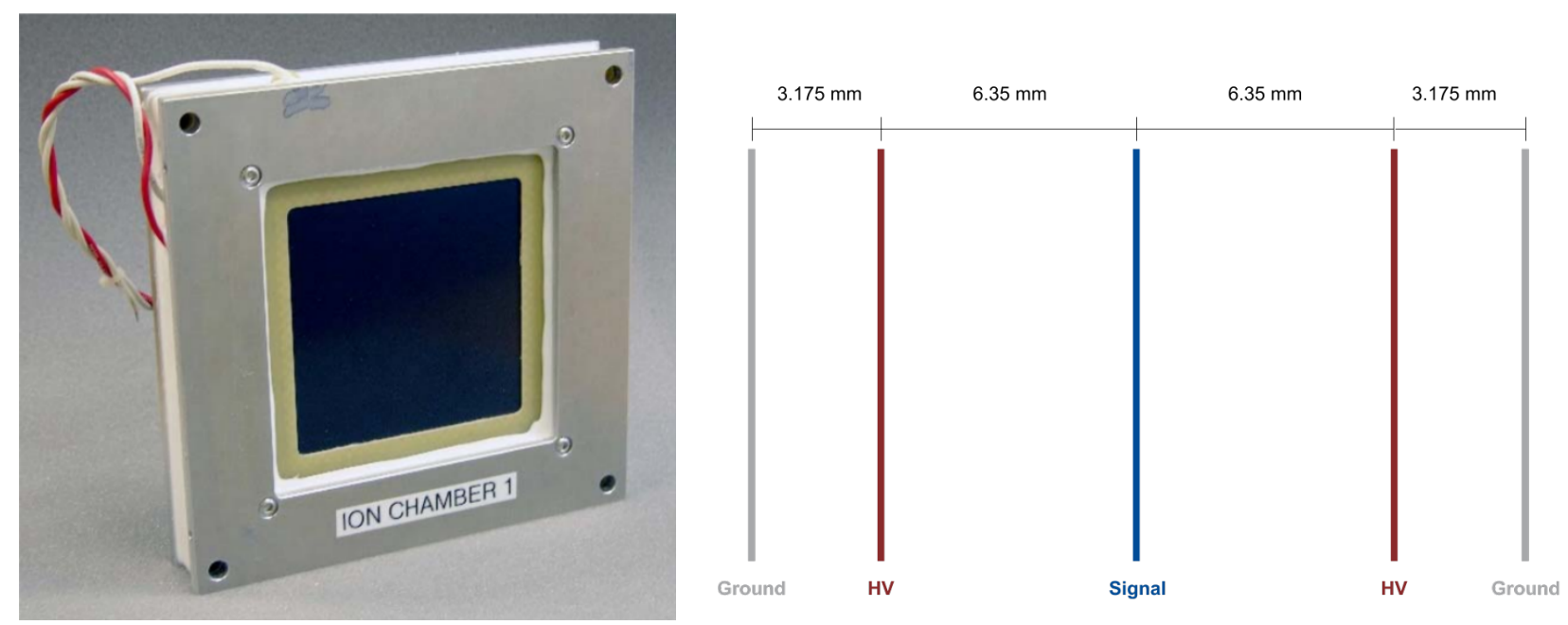

Figure 3. A photograph of a Schoo-style IC (left), and diagram showing the high-voltage and signal foil spacing (right).

is of the newest design created by Daniel Schoo and built at Fermilab. An excellent description of these chambers is found in Dan's digitizer manual. ${ }^{1}$ A photograph of one such IC is pictured in Figure 3, as well as a diagram showing the spacing between HV and signal foils. The digitizer electronics that measure ionization current are connected such that the signal foil represents a virtual ground. Traditionally, the high-voltage supply used with these chambers applies a negative voltage to the HV planes, so the particles collected on the signal plane are positive ions. This high-voltage has typically been - $800 \mathrm{~V}$, and the 
chamber is filled with a gas mixture of $80 \%$ Argon and $20 \% \mathrm{CO}_{2}$. The gas pressure in the chamber is kept slightly higher than atmospheric pressure to allow gentle gas exchange over long periods of time.

\section{Digitizer Modules}

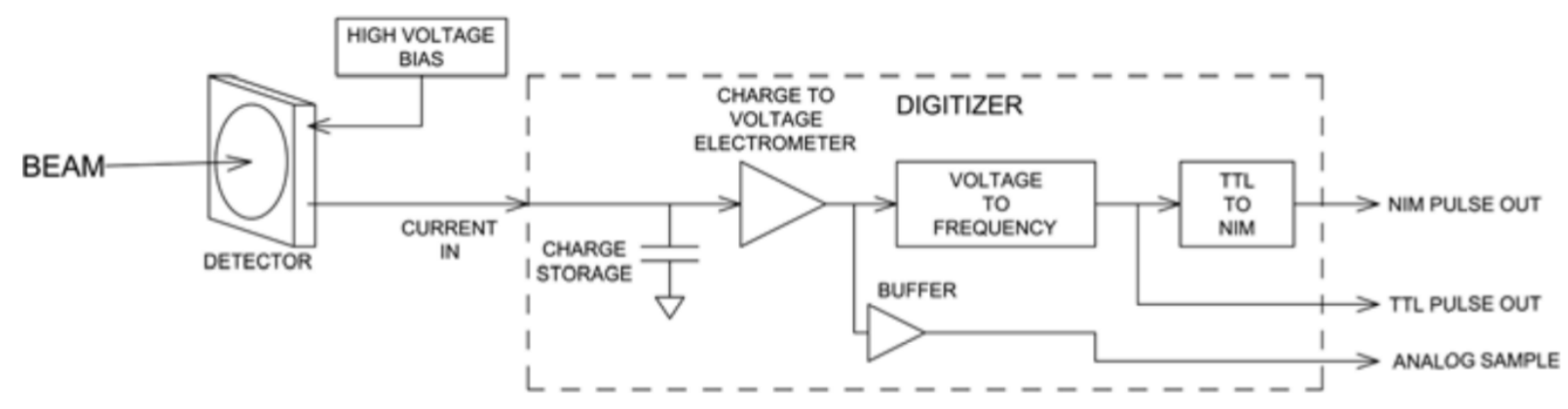

Figure 4. A block diagram overview of the Schoo digitizer module. ${ }^{1}$

The electronics module that measures and digitizes the ionization current is also designed by Daniel Schoo and built at Fermilab. ${ }^{1}$ A block diagram representation of the module is shown in Figure 4. The digitizer first converts the small ionization current from the IC signal foil to a voltage using an electrometer amplifier. The voltage output of the electrometer is then converted to a NIM or TTL standard pulse train using a voltage-to-frequency ("VFC") converter; this pulse train is counted by an external module to provide a high-resolution and dynamic range digitized count that is proportional to the total integrated ionization current during the beam pulse. A capacitor in parallel with the electrometer input provides noise isolation, high-frequency filtering, and charge storage to provide a buffer so beam intensity information is not lost if the VFC is unable to digitize quickly enough. Thus a potential source of saturation at high beam intensity is the VFC chip. The maximum output of the chip is $100 \mathrm{kHz}$, and without the charge-storage capacitor at the electrometer input, some signal is lost at high intensity once the required pulse rate exceeds $100 \mathrm{kHz}$. The input capacitor mostly alleviates this issue by storing excess charge if the VFC is output at maximum rate, then discharges to the VFC once the rate decreases. Thus the total number of pulses from the module should be read by the control system a minimum of 10 characteristic time-constants after end-of-spill. In Switchyard, the $\$ 37$ clock event 2 seconds after end-of-spill is traditionally used to measure integrated beam intensity, so a maximum characteristic time constant of $200 \mathrm{~ms}$ is acceptable.

\section{Digitizer Saturation}

Saturation of the VFC can also be avoided by adjusting the gain of the electrometer portion of the circuit; as long as the output of the electrometer does not exceed $10 \mathrm{~V}$, the VFC will not be required to exceed its maximum output rate to keep up with the incoming beam signal. Thus it is important to adjust the electrometer gain to fit the application of the digitizer. By default, the digitizer gain is 500 counts per nanoCoulomb of integrated beam charge in the IC. For high-intensity applications, a gain of 1 count $/ \mathrm{nC}$ was required to prevent VFC saturation. Figure 5 shows the circuit schematic for the electrometer portion of the digitizer and highlights the feedback resistor that determines the gain.

To prevent digitizer saturation at up to $1 \mathrm{E} 13 \mathrm{ppp}$, the default $50 \mathrm{MOhm}$ resistor was changed to $100 \mathrm{kOhm}$ to achieve the 1 count/nC reduced gain. Figure 6 shows beam data comparing the high-gain and low-gain digitizers; it is clear that the reduced gain of 1 count/nC greatly improve the response linearity at higher beam intensity. The upper intensity limit before nonlinear roll-off for the lower-intensity digitizer is much higher, around 2E12 protons per pulse. Note from Figure 6 that non-linear roll-off persists, though it is now not due to digitizer saturation, because the analog voltage is well below 10V. This implies another saturation mechanism at high beam intensity.

\section{lon Clearing Voltage}

Zwaska et. al. have shown ${ }^{3}$ that at very high beam fluence, the buit-up electron/ion pairs in ICs create a space-charge-induced electric field that partially cancels the clearing field in the chamber. This partial cancellation slows the effective drift velocity of the electron/ion pairs, which increases the chance of recombination in transit to the electrodes. This "recombination loss" results in reduced signal at higher intensity, which could explain the aforementioned "roll-off" in response. 


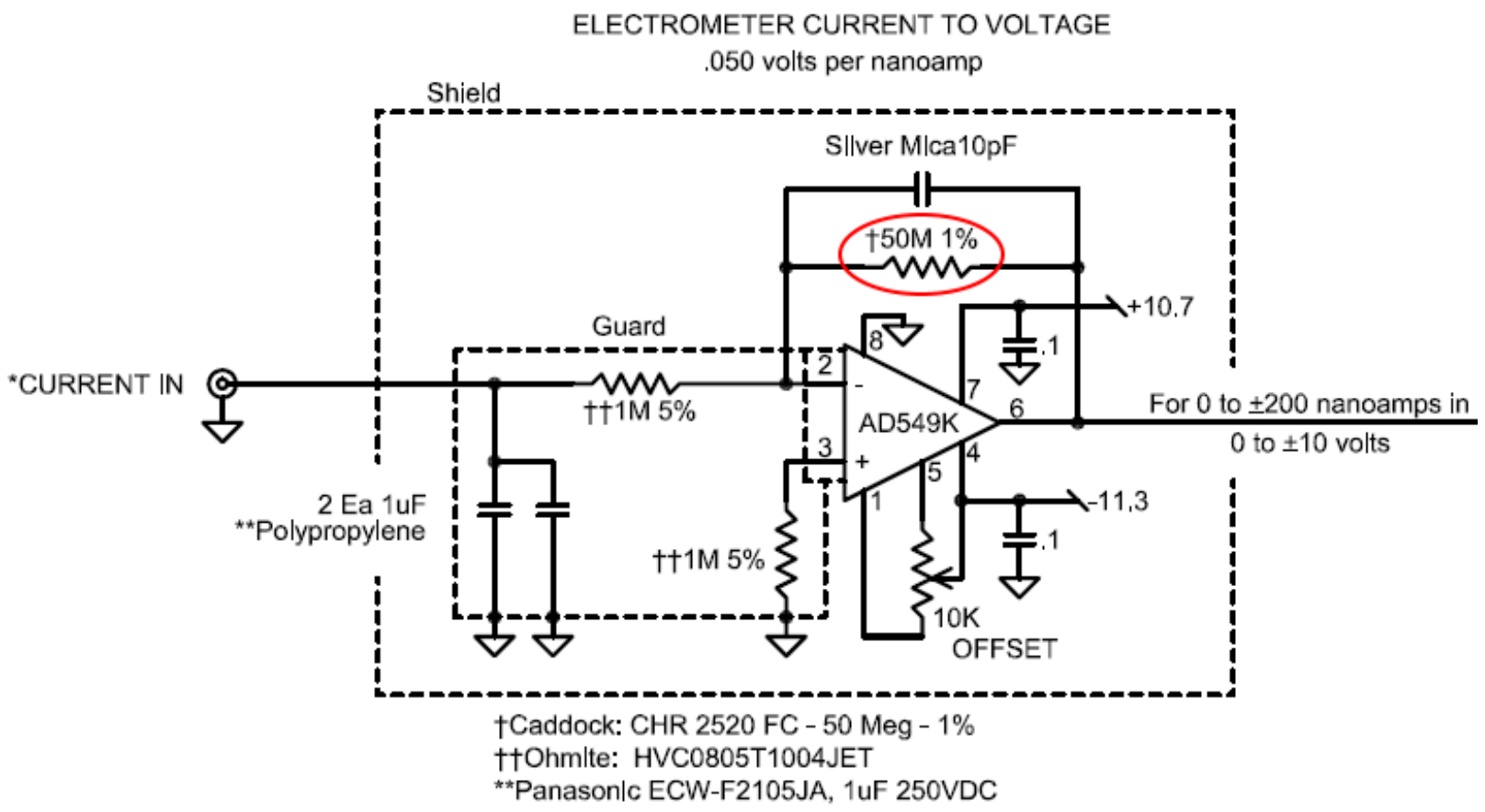

Figure 5. Circuit diagram for the electrometer portion of the digitizer. The red circle indicates the resistor that determines the gain. ${ }^{1}$
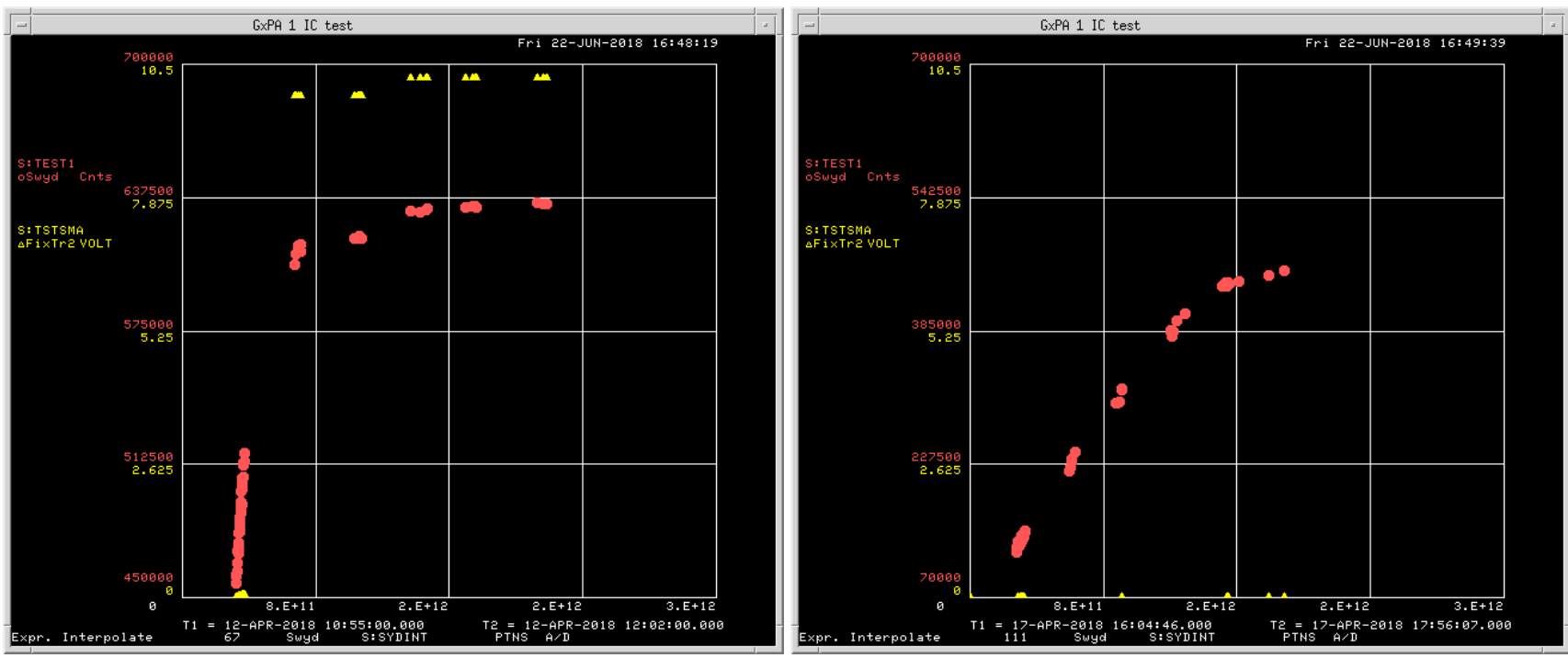

Figure 6. Comparison of ion chamber response (red points) for $500 \mathrm{c} / \mathrm{nC}$ gain (left) and $50 \mathrm{c} / \mathrm{nC}$ gain (right). Analog output (yellow points) above $10 \mathrm{~V}$ indicates digitizer saturation. Response roll-off point is noticeably higher for reduced gain, and analog output shows digitizer is no longer in saturation.

The remedy for recombination loss is to operate the IC at high clearing voltage, and thus it is necessary to explore the voltage plateau curve of the test chamber. In general, high-fluence ICs need the highest-attainable voltage in the "plateau" region of the curve without causing breakdown in the gas. Figure 7 shows the voltage plateau curves for the test IC in positive and negative polarities for consistent 5E11 protons per pulse. The negative power supply was limited by design to $-2500 \mathrm{~V}$ max, which defines the upper-end of the negative polarity curves; the positive supply is capable of $10 \mathrm{kV}$, but breakdown occurred well before that limit was reached.

There appears to be no discernible difference between polarities in terms of the voltage plateau curve, and both show the 

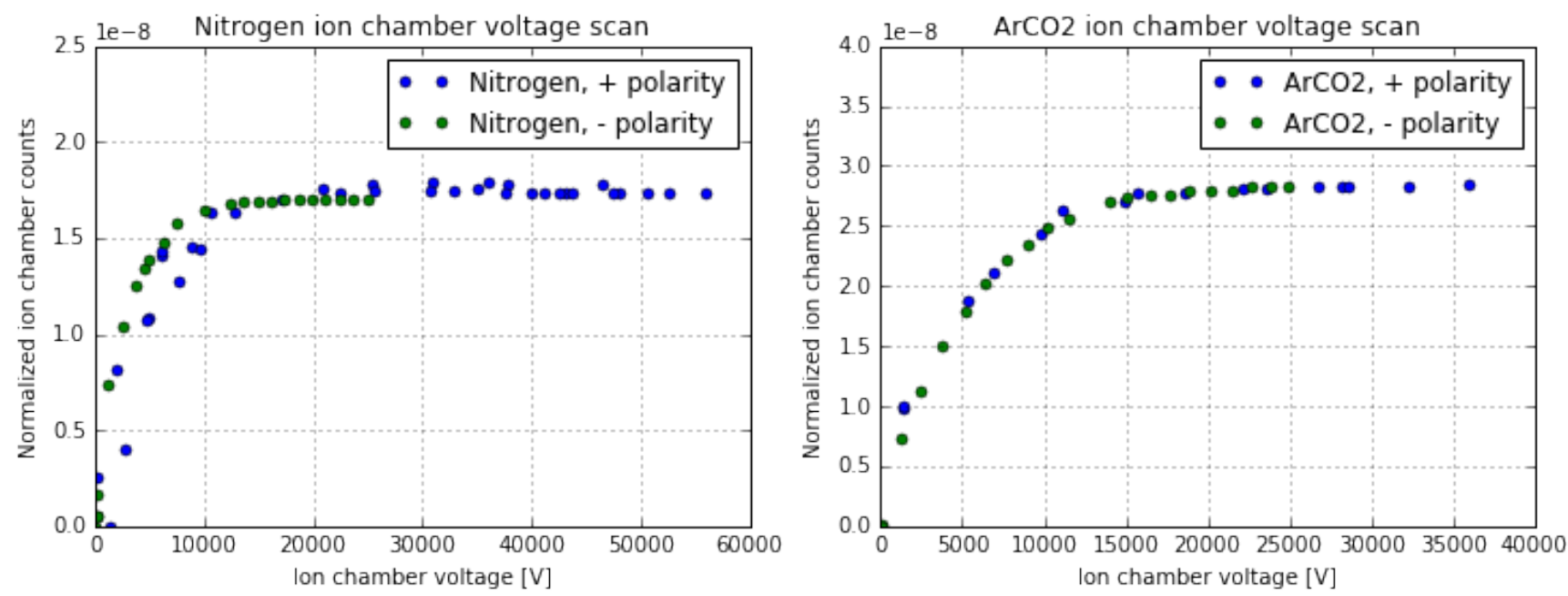

Figure 7. Ion chamber response for consistent beam intensity as a function of ion clearing voltage for both Nitrogen gas and standard $80 \%$ Argon 20\% CO2 mix.

ability for the IC to operate at significantly higher clearing voltage than the traditional $800 \mathrm{~V}$. For remaining high-fluence beam tests, a clearing voltage of $+/-2500 \mathrm{~V}$ was chosen to allow for symmetric testing of the IC response for both polarities. Figure 8 and Figure 9 show the results of a beam intensity scan with $+/-2500 \mathrm{~V}$ clearing potential for both Nitrogen and standard $80 \% / 20 \% \mathrm{ArCO}_{2} \mathrm{ix}$.
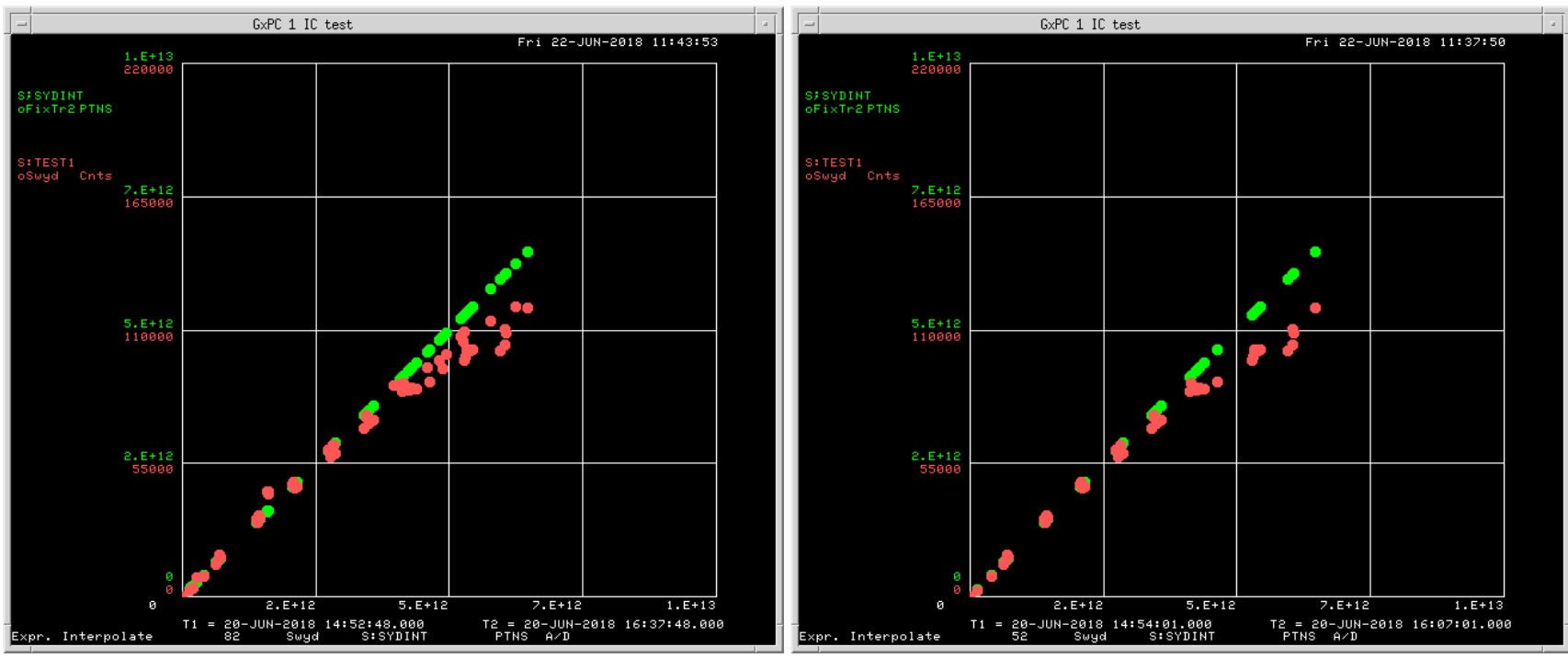

Figure 8. IC intensity scans with $\mathrm{ArCO}_{2}$ standard $80 \% \mathrm{Ar} 20 \% \mathrm{CO} 2 \mathrm{mix}, 1$ count/nC sensitivity digitizer, and -2500 V (left) and $+2500 \mathrm{~V}$ (right) clearing potential. The IC response is in red, and the response of reference SEM in the same area is in green.

The results of these beam scans show a clear improvement in high-intensity linearity of the IC response with clearing voltage of $+/-2500 \mathrm{~V}$ as compared to the $800 \mathrm{~V}$ scans in Figure 6. There appears to be no discernible difference between positive and negative polarity of the clearing voltage. However, it is clear that Nitrogen gas performs much better than $\mathrm{ArCO}_{2} \mathrm{thigh}$ beam intensity; even at $+/-2500 \mathrm{~V}$ clearing voltage, there remains some response roll-off with $\mathrm{ArCO}_{2}$ as about about 4E12 protons per pulse.

Figure 10 and Figure 11 show a quantitative analysis of the response linearity for both $\mathrm{ArCO}_{2}$ nd Nitrogen at $+2500 \mathrm{~V}$. The estimated error in the data, shown in a grey band, comes from the systematic error reported by the Fermilab counting lab when SEMs and ICs are calibrated using copper foil irradiation. An estimated systematic error of about $8 \%$ is typical 

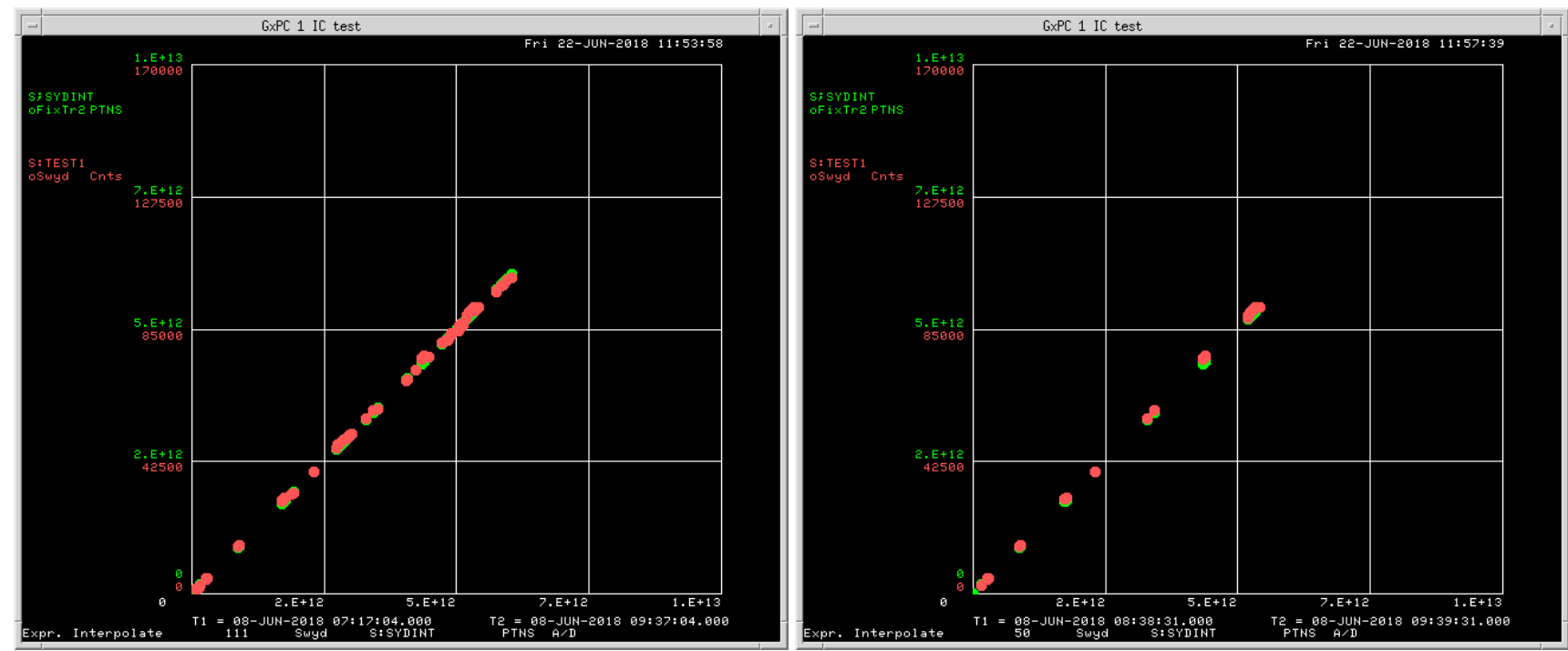

Figure 9. Ion chamber intensity scans with Nitrogen, 1 count/nC sensitivity digitizer, and $-2500 \mathrm{~V}$ (left) and $+2500 \mathrm{~V}$ (right) clearing potential.

for such calibrations, so the grey error band boundaries are determined by a linear fit to the data plus or minus the estimated systematic error and counting noise. The normalized fit residual for each data point is computed as the difference between the data and the fit divided by the error band half-width. Comparing Figure 10 and Figure 11, it is clear that the IC with Nitrogen is dramatically more linear up to $6 \mathrm{E} 12 \mathrm{ppp}$ compared to $\mathrm{ArCO}_{2}$
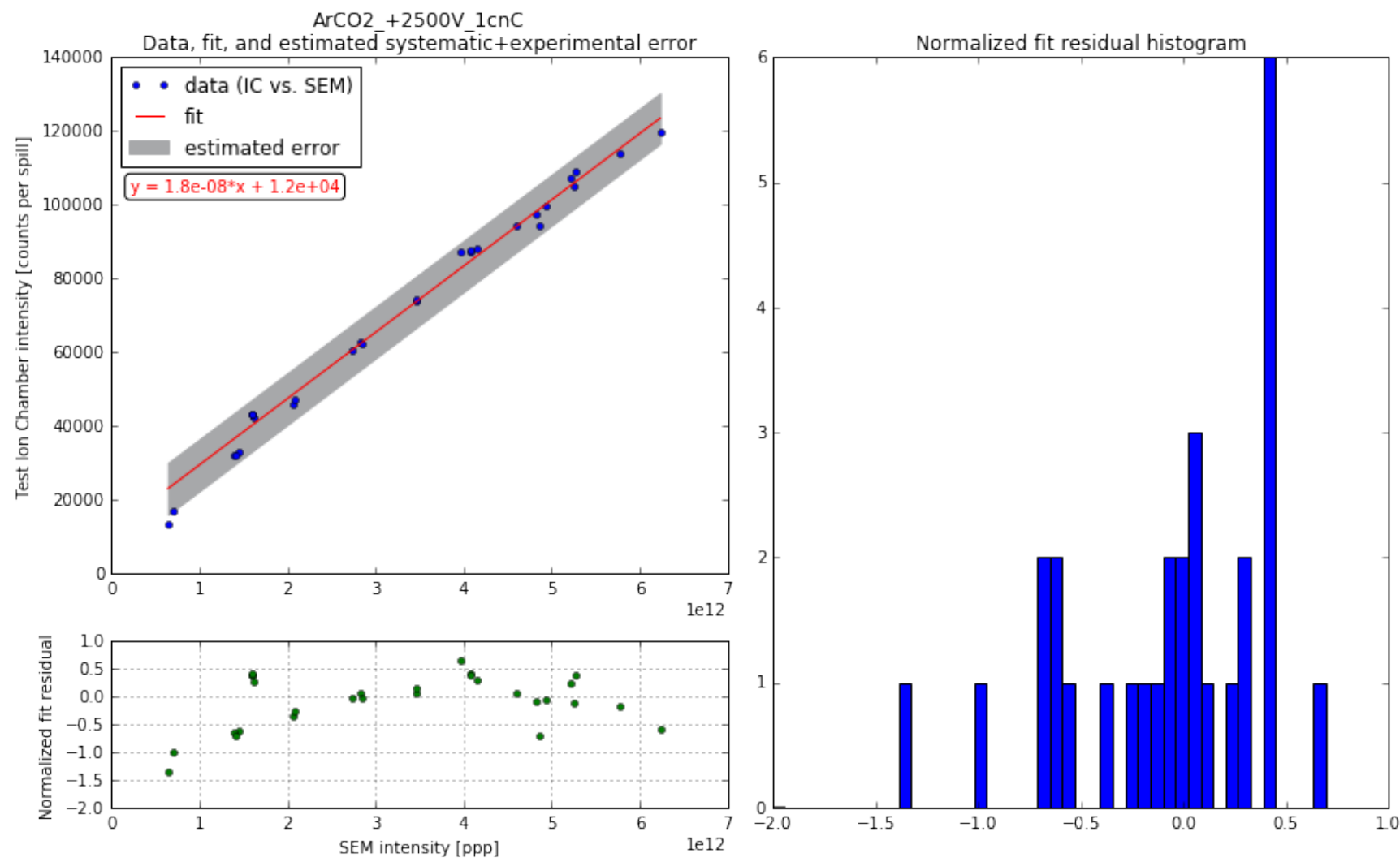

Figure 10. Ion chamber response for consistent beam spot size as a function of protons per pulse for standard $80 \%$ Argon $20 \%$ $\mathrm{CO} 2 \mathrm{mix}$ at $+2500 \mathrm{~V}$. Estimated error band comes from the $8 \%$ estimated systematic error in absolute foil irradiation calibration. 

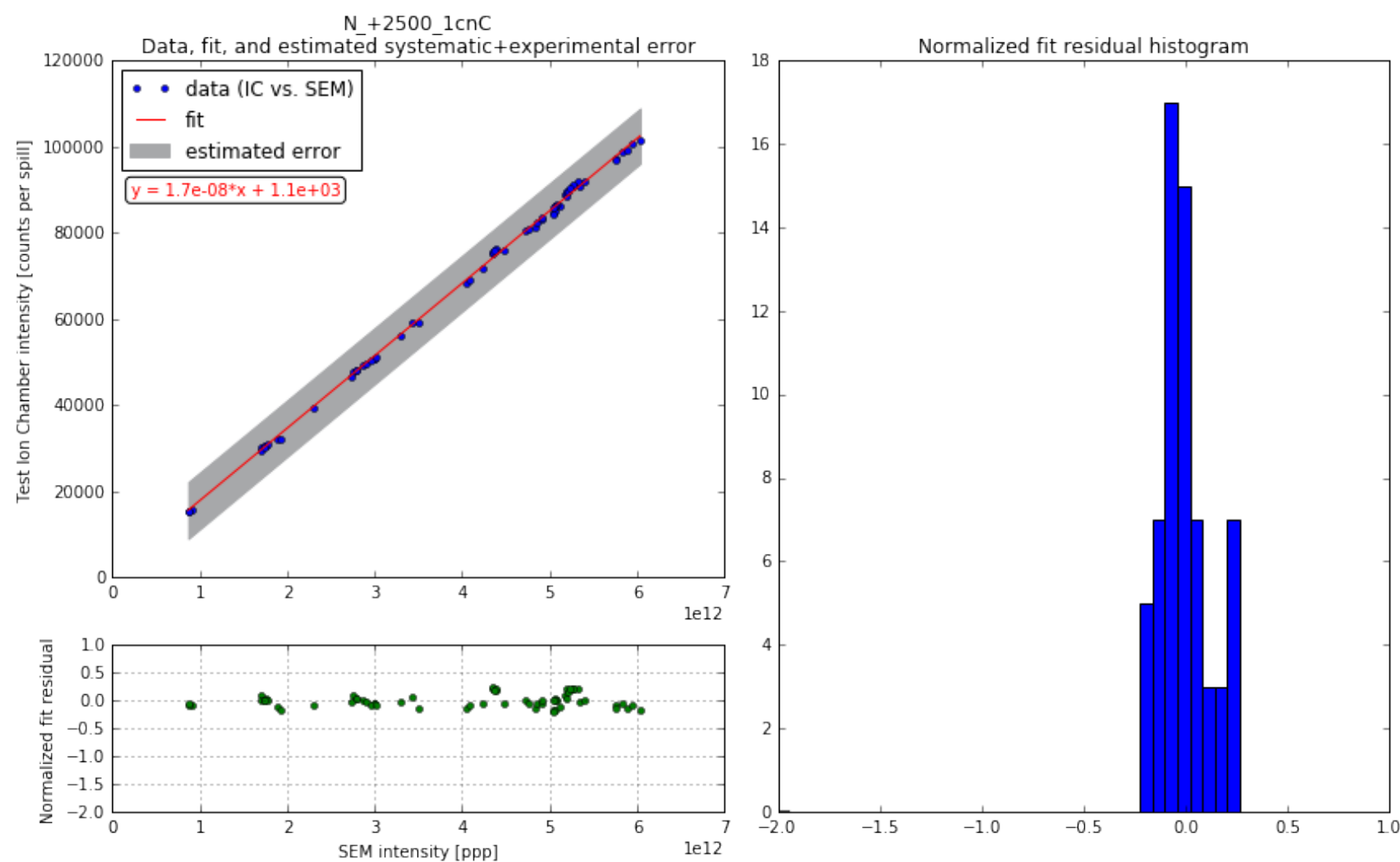

Figure 11. Ion chamber response for consistent beam spot size as a function of protons per pulse for Nitrogen gas at $+2500 \mathrm{~V}$. Estimated error band comes from the $8 \%$ estimated systematic error in absolute foil irradiation calibration.

\section{Conclusions}

Fermilab-built ICs and digitizers have been tested for high beam intensity, and several sources of non-linear saturation have been identified and mitigated. Significant increase in linear response up to 6E12 ppp has been achieved by reducing the digitizer sensitivity, switching from $\mathrm{ArCO}_{2} \mathrm{o}$ Nitrogen gas, and increasing the clearing voltage to $2500 \mathrm{~V}$. With these modifications, it has been shown that available equipment is capable of replacing costly SEMs for beam intensity monitoring in many of the SY120 beamlines.

\section{References}

1. Ultra high input impedance electrometer / pulse train converter for the measurement of beam intensity current digitizer ii. Daniel Schoo, Accelerator Division Beams Document 6494-v1: http://beamdocs. fnal.gov/AD-public/ DocDB/ShowDocument?docid=6494.

2. Low mass multiwires. Gianni Tassotto, Accelerator Division Beams Document 6874-v1: https://beamdocs. fnal. gov/AD-public/DocDB/ShowDocument?docid=6874.

3. Zwaska, R. M. et al. Beam tests of ionization chambers for the numi neutrino beam. In 2002 IEEE Nuclear Science Symposium Conference Record, vol. 1, 645-649 vol.1 (2002). 